\title{
DETERMINANTES DE LA FUNCIÓN CONTABLE
}

\section{Diego Delgadillo $R$.*}

Fuera de las cuestiones técnicas, la percepción es la parte más importante del pensamiento, aunque, tal vez, la educación tradicional no nos ha dado esa impre-

sión. La mayor parte de los errores de juicio se deben a errores de percepción.

Edward de Bono, 1997

En años recientes, a la par del cuestionamiento sobre el carácter del conocimiento contable, se havenido dando una serie de fenómenos, no tan perceptibles quizás, alrededor del rol que los contables desempeñan en la empresa.

Los cambios económicos, fácilmente observables por los académicos y entendidos, permiten aseverar con relativa certeza que las empresas están fuertemente obligadas a replantear cada una de las viejas maneras de ver su marcha de cara a la formulación de nuevas estrategias competitivas y de supervivencia.

De igual manera, en la profesión contable los cuestionamientos se hacen reiteradamente acerca de la naturaleza y del carácter del conocimiento contable. Los planteamientos siguen refiriéndose a la función de la contabilidad y a los problemas que ella soluciona, a sus limitaciones, a etc.

Este trabajo toma el camino de la reflexión para hacer explícitos tres aspectos que vienen a constituirse en hitos importantes para la demarcación de la función contable del presente y de los próximos años.

- Profesor de la Facultad de Administración. 
Se busca con ello contribuir en el debate continuo de los académicos de la contabilidad y, en general, de la profesión de la Contaduría Pública.

Es importante y necesario, desde el aula de clase, maximizar los esfuerzos por reconstruir las bases conceptuales que determinan la función de dirección y de organización de los sistemas de información contable.

Las premisas en las que se basa esta propuesta son las siguientes:

Primera; El cambio en la legislación contable colombiana que introduce a partir de 1993 el enfoque o paradigma de la utilidad de la información contable para la toma de decisiones de carácter económico.

Segunda: Las nuevas demandas a la contabilidad provenientes de los gerentes y administradores de empresas con entornos cada vez más competitivos que interrogan constantemente acerca del valor agregado de la contabilidad para administrar sus empresas.

Tercera: La necesidad de definir en forma precisa las funciones del director del área contable de la empresa con base en una caracterización de la contabilidad como sistema de información contable.

\section{EL CAMBIO EN LA LEGISLACIÓN CONTABLE: INTRODUCCIÓN DEL PARADIGMA DE LA ÚTILIDAD DE LA INFORMACIÓN CONTABLE}

En las décadas de los años cuarenta y cincuenta era ya común en la literatura contable norteamericana la expresión de que la contabilidad tenía como objetivos el cálculo de la utilidad y la valuación de los activos de la empresa, además, consideraba importante el enfoque de la ganancia líquida realizada. Aún, en la década de los años ochenta, algunos autores fundamentaban las técnicas contables a partir de la definición de la contabilidad como el arte del registro de las transacciones de la empresa.

A finales de los años sesenta, tanto en los Estados Unidos de América como en Inglaterra, los gremios profesionales luego de esfuerzos en investigación, comenzaron a trabajar en la formalización de nuevas propuestas que hicieran explícita la misión y objetivos de la contabilidad 
financiera. En este sentido, dos trabajos con enfoque coincidente son los que han guiado buena parte del estudio de los profesionales de la contabilidad a partir de ese entonces: el informe Trueblood del Instituto Americano de Contadores y el Corporate Report en Inglaterra.'

Es conocido que en Colombia se empezó a hablar con fuerza de ley sobre normas contables a partir de 1986 y que ha venido consolidándose un cuerpo de profesores y estudiosos de la teoría contable a fin de fundamentar y normalizar las técnicas y los procesos contables en los distintos sectores de la economía. En ese mismo sentido, se han conformado organísmos como el Consejo Técnico de la Contaduría que, de una u otra manera, han dado los primeros pasos en la normalización contable. Estos esfuerzos y otros factores condujeron a una pronta reforma del marco conceptual aprobado eseaño y desembocaron en unamás ampliaconcepción del marco de referencia para el trabajo de la contabilidad comercial: el Decreto 2649 de 1993.

Este acto legislativo introdujo el paradigma de la utilidad de la información contable, haciendo explícitos los objetivos de la contabilidad y de la información contable con base en las necesidades de información de los usuarios para tomar decisiones de naturaleza económica.

¿Cuáles son las consecuencias del paradigma de la utilidad incorporado como norma en este acto legislativo?

La respuesta la tiene en forma precisa el profesor Jorge Túa P. quien manifiesta al respecto lo siguiente:

- La progresiva ampliación del concepto de usuarios de la información financiera.

- La búsqueda y discusión, promovida por esta óptica utilitaria, de los objetivos y requisitos de la información financiera

- Su incidencia en la cantidad de información suministrada o, al menos, requerida a la empresa.

\footnotetext{
' Belkaoui Ahmed. Accounting Theory. Harcourt Brace Jovanovich, 1981
} 
- Su influencia en la evolución de la epistemología contable.

- Su incidencia en el desarrollo de la investigación empírica en nuestra disciplina. $^{2}$

A pesar de la importancia de las implicaciones que se han presentado a partir del surgimiento del mencionado paradigma, para los propósitos de este informe es necesario resaltar aquí los tres primeros.

Los usuarios de la información han tomado posicionamiento y jerarquía, es decir, ellos se constituyen en el cliente por excelencia de los productos que emanan del sistema contable. Es importante, como consecuencia, situarse frente a ellos, caracterizarlos, conocer sus actuaciones, el medio en que se desenvuelven, estudiar sus necesidades informativas y satisfacer sus demandas. La contabilidad así tomada, dejó de ser un mero registro de carácter legal, para convertirse en un instrumento necesario de la gestión los administradores. Este carácter de uso de la información contable, si bien se sobreentendía, se vuelve ahora más explícito.

Por otra parte, las funciones tradicionales de la contabilidad de calcular la utilidad y efectuar la valuación de los recursos del ente económico, no solamente se enriquecieron sino que se ampliaron con la formulación de que la contabilidad y la información contable se debían preparar y presentar como una base importante para tomar decisiones.

Ligado a este enfoque de fijación precisa de objetivos y con el objeto de darle más coherencia al marco de referencia, la legislación contable colombiana permite ahora la valuación y representación de los recursos de la empresa y de los hechos económicos que los afecten, no únicamente a su valor de costo, sino que abrió las compuertas a otro tipo de metodología o base de valuación que incluye el valor actual o de reposición, el valor de realización o de mercado y el valor presente o descontado.

El mismo concepto de causación contable tradicional tuyo que ser replanteado para fortalecer con fuerza el nuevo de realización económica

${ }^{2}$ Belkaoui Ahmed. Accounting Theory. Harcourt Brace Jovanovich, 1981 
a fin de hacerlo más congruente con las demás guías para la aplicación contable.

Éste es un panorama muy alentador para los profesionales de la contabilidad y de la auditoría, pues, permite mayor libertad en su actuación y aleja a la contabilidad de la posibilidad de ser un proceso de instrumentación técnica y, como ya se mencionó anteriormente, de ser un requisito exclusivamente de carácter legal y esencial de la tributación.

Sin embargo, es necesario precisar desde otro ángulo de observación que el nuevo enfoque demanda, igualmente, una mayor atención en el manejo de la contabilidad dentro de la empresa.

Los contadores que han actuado bajo el enfoque de la ganancia líquida realizada y con la concepción del "arte del registro de transacciones", deben ampliar su campo de observación, reestructurar su percepción de la contabilidad y actuar en función de una mayor jerarquía para el área contable y bajo la premisa de que el manejo contable no solo demanda conocimientos exclusivamente de carácter contable, sino de naturaleza administrativa y económica con el fin de construir un perfil para la dirección contable que facilite la satisfacción de las demandas calificadas de los usuarios de la información contable. La contabilidad tiene sentido, no por sí misma, sino por su contenido y relación con el entorno que la afecta.

\section{LAS NUEVAS Y ANTIGUAS SOLICITUDES DE INFORMACIÓN CONTABLE PARA ADMINISTRAR: VALOR CONTABLE AGREGADO}

Al examinar los objetivos planteados en el nuevo paradigma, es preciso señalar que son coherentes y naturales con respecto al antiguo oficio de dirigir y manejar las entes y organizaciones productivas. Posiblemente se hacen explícitos y se precisan los informes que se deben suministrar. La contabilidad siempre se justificó contra un posible caos informativo dentro de la empresa. Pero la pregunta es ¿por qué ahora, al menos en lo que concierne al caso colombiano? 
Parte de la respuesta es, sin duda, que el país ha reformulado ostensiblemente sus esquemas tradicionales de trabajo en el sector financiero y modernizado el aparato y los procesos tributarios, para mencionar únicamente estos dos casos. Durante la década de los años ochenta fueron necesarias varias reformas a la legislación que regulaba las transacciones y operaciones financieras. La Ley 45 de 1990 es una muestra evidente, a la par de la normalización en la revelación financiera del mismo sector. Los procesos de tributación han sido cada vez más eficientes y más ágiles frente a los tediosos requisitos que el contribuyente debía cumplir anteriormente. Estas muestras de cambio han sido deliberadamente concebidas como una resultante de la gran presión de la dinámica empresarial y, en general, del sistema económico, así como el papel que ejercen las agencias reguladoras del Estado. Hay casos, como el del sector financiero, que recibieron gran empuje a raíz de su propia crisis generada con énfasis en 1992.

Por otra parte, el sistema económico en general, habiendo globalizado cada vez más los mercados, ha presionado a las empresas a ser más eficientes y abrirse a una competencia más fuerte, por lo que sus estrategias de negocios, así como sus herramientas administrativas deben estar siempre monitoreadas a fin de que se constituyan en un factor proactivo con vista para lograr la misión y objetivos propios. Elárea contable no está ajena a estos requerimientos. Como cualquier otraárea de responsabilidad administrativa de la organización, debe mostrar una relación beneficio/ costo positiva, de modo que no sea una carga necesaria simplemente para cumplir unos requisitos legales, sino que debe proveer un valor agregado a la empresa y llegar a convertirse en un factor competitivo. Es necesario recordar que toda empresa que haya crecido y tenido éxito debe haber superado los problemas de información contable propios. Un distintivo de una empresa exitosa debe ser su área contable bien administrada.

Pero ¿qué es valor contable agregado?

Es la contribución positiva que el área o sistema de información contable, produce en logro de la misión de la empresa y la satisfacción de los usuarios contables. Pero ¿en qué consiste esa contribución? 
La contribución efectiva del sistema contable es el significado que la información contable financiera posee y que le confiere valor de uso en el momento de decidir sobre cualquier asunto relacionado.

El sistema contable debe producir información significativa, convalorde uso. Entre más atributos y más cualidades tenga la información contable, mayor será su utilidad y su significado para los usuarios, es decir, tendrá más valor. Con ello, en el trabajo de diseño del sistema contable, un paso importante es la definición de las cualidades y atributos del producto que se quiere fabricar o producir en el área contable. La pregunta para el contador es, ¿cómo manufacturar información contable con significado?

El significado se produce, si se concibe y caracteriza en forma debida el sistema de información contable a fin de que se diseñen sistemas operativos contables que interpreten y concreten la naturaleza del ente económico, en particular, y, finalmente, para que produzcan información coherente y más cercana a su realidad.

La concepción del sistema implica definir al menos los siguientes elementos:

Primero : El entorno propio en que se desarrolla.

Segundo: La misión y objetivos del sistema.

Tercero : Loscomponentes.

Cuarto : Sus recursos.

Quinto : Su administración.

\section{LA REDEFINICIÓN DE LA FUNCIÓN DIRECTOR DEL ÁREA CONTABLE DE LA EMPRESA: \\ EL ADMINISTRADOR DE UN SISTEMA DE INFORMACION}

Observados los nuevos desarrollos teóricos y las presiones del entorno empresarial que redefinen el papel de la contabilidad y de la información contable, se deben, como consecuencia lógica, esbozar las pautas para el papel que el Contador, como director del área contable, debe desempeñar. 
Olvidados los viejos esquemas de trabajo, se hace obligada la modernización de la función contable considerando, entonces, que la producción de informes contables útiles para administrar no puede improvisarse y que el producto final debe reunir la calidad de ser pertinente y oportuno, confiable e íntegro. Luego, el nuevo papel del Contador directivo implica desarrollar una función de triple carácter:

En primer lugar, administrar los recursos a cargo, es decir, los que se incorporan en el área contable siguiendo las pautas racionales, con base en los fundamentos de las escuelas del pensamiento administrativo, así como las técnicas administrativas e instrumentación disponibles, de manera que se efectúe el proceso de producción de información contable de manera eficiente.

En segundo lugar, revelar la información financiera, con base en las políticas y pautas preestablecidas.

Y en tercer lugar, asesorar y servir de consultor de los usuarios de la información contable, tanto internos como externos.

La administración del área contable es un trabajo que comprende el desarrolloy despliegue de esfuerzos sistemáticos para cumplir las funciones de Planeación, Organización, Dirección y Control, asícomoel análisis de situaciones con escenarios complejos; decidir y comunicar las decisiones.

La producción de información es una función de transformación de datos en informes significativos. Implica la adecuación más productiva de los recursos disponibles personas, medios materiales y financieros- para cumplir la misión contable, teniendo como base tanto los elementos de política contable emanados de la dirección del ente económico, así como la naturaleza de sus procesos operativos y, por su puesto, el marco de referencia que proporcionan la teoría y técnicas contables y financieras.

La asesoría y consultoría a los usuarios de la información contable, por parte del director contable se puede considerar como una función natural porque, una vez se suministre, se precisa de la sustentación, de la explicación, de la interpretación y de la síntesis. Ésta es una función complementariaa la de la revelación financiera en la empresa. Es el primer 
paso en el camino de la toma de decisiones por parte de los responsables de la dirección de la empresa.

Pero qué ¿queda de estas consideraciones para la universidad y, en especial, para los docentes?

Al menos dos cosas:

Primera: Replantear sistemáticamente los esfuerzos en el proceso de enseñanza-aprendizaje a fin de que la comunidad contable vaya adquiriendo una noción más precisa del trabajo de dirección contable acorde con las necesidades actuales de la empresa.

Segunda: Concientizar a los futuros profesionales de que no solo de contabilidad viven los contadores, sino que, además, es preciso demarcar más ampliamente los límites de nuestra labor profesional a fin de hacerla más congruente con el mundo circundante, mediante un esfuerzo continuo para lograr un conocimiento del entorno en que se usa la información contable y en el que es dada la facultad de certificar la realidad económica mediante la fe pública.

\section{BIBLIOGRAFIA}

BELKAOUI Ahmed. Accounting Theory. HBJ Inc. New York, 1981.

CHURCHMAN WEST. El Enfoque de Sistemas. Edit. Diana. México, 1978.

MARTÍNEZ F. Carlos E. Administración de Organizaciones. Teoría y Práctica. U. Nal. de Col. Fac. de Ciencias Ec. 1989.

Túa P. Jorge. Lecturas de teoría e investigación contable. Cijuf. Medellín. 1995. 